\title{
A functional form for the energy spectrum parametrizing bottleneck and intermittency effects
}

\author{
Johan Meyers ${ }^{1, a)}$ and Charles Meneveau ${ }^{2, b)}$ \\ ${ }^{1}$ Department of Mechanical Engineering, Katholieke Universiteit Leuven, \\ Celestijnenlaan 300A-bus 2421, B3001 Leuven, Belgium \\ ${ }^{2}$ Department of Mechanical Engineering, Johns Hopkins University, \\ 3400 North Charles Street, Baltimore, Maryland 21218, USA
}

(Received 29 January 2008; accepted 29 April 2008; published online 24 June 2008)

\begin{abstract}
A functional form for the energy spectrum is proposed, which includes a predissipative bottleneck effect and an intermittency correction. The form contains five parameters for which five constraints are formulated. Three of them are related to the condition that the integrals of the spectrum, $E(k)$, $k^{2} E(k)$, and $k^{4} E(k)$, should yield appropriate values for total energy, enstrophy, and derivative skewness. Two extra conditions are inferred from the location and height of the intermittency corrected dissipation peak, which is found to be independent of Reynolds number $\left(70<\operatorname{Re}_{\lambda}\right.$ $<1200$ ) in high-resolution direct numerical simulations. Good representation of Direct Numerical Simulation (DNS) and experimental data is found for a range of Reynolds numbers $\operatorname{Re}_{\lambda}$ between 250 and 1200. (C) 2008 American Institute of Physics. [DOI: 10.1063/1.2936312]
\end{abstract}

\section{INTRODUCTION}

Over the years, several proposals for a functional form to describe the energy spectrum $E(k)$ of high Reynolds number turbulence have been proposed. Renewed current interest in accurate and practical representations of energy spectra stems, among others, from validation studies of large eddy simulation (LES) models. ${ }^{1,2}$ These require detailed comparisons of LES results with the best representations of "true" energy spectra. The inclusion of finite Reynolds number effects makes accurate representations of energy spectra across all scales highly desirable. Most of the early attempts concentrated on the dissipation range of the spectrum, defining a high-wavenumber "damping function" to merge the inertialrange $k^{-5 / 3}$ spectral slope to the viscous-dominated region. The Heisenberg, Pao, and Kraichnan spectra ${ }^{3}$ are wellknown classic approaches. Currently, the Kraichnan ${ }^{4}$ exponential decay of the energy at very high wavenumbers $[E(k) \sim \exp (-\alpha k)]$ is believed to be the most appropriate representation at high wavenumbers. ${ }^{5}$

Later, Smith and Reynolds ${ }^{6}$ have shown that functional forms for the energy spectrum should comply with a set of basic relations, i.e., the total enstrophy

$$
\mathcal{E}=\int_{0}^{\infty} k^{2} E(k) d k
$$

(with $\mathcal{E}=\left\langle|\boldsymbol{\omega}|^{2}\right\rangle / 2$, where $\boldsymbol{\omega}=\nabla \times \mathbf{u}$ is the vorticity and $\mathbf{u}$ is the velocity field), while the total palinstrophy,

$$
\mathcal{P}=\int_{0}^{\infty} k^{4} E(k) d k
$$

(with $\mathcal{P}=\frac{1}{2}\left\langle|\nabla \times \boldsymbol{\omega}|^{2}\right\rangle$ ) is also important since it can be related to the skewness of the longitudinal velocity derivative,

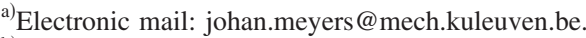

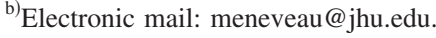

$\mathcal{S}_{3}$, when enstrophy production and dissipation are in equilibrium. ${ }^{2,6}$ Interestingly, however, in Ref. 6, it was demonstrated that satisfactory values of $\mathcal{S}_{3}$ cannot be attained by only using exponential decay of the spectrum at high wavenumbers. Attempts to improve this by shifting the onset of the exponential decay to higher wavenumbers ${ }^{7,8}$ are only partially satisfactory and fail to yield realistic values of $\mathcal{S}_{3}$ at finite Reynolds numbers. ${ }^{2}$

Experiments and Direct Numerical Simulation (DNS) results over the past decade confirm the existence of a bottleneck at the onset of the dissipation range, which appears as a bump in the compensated spectrum. ${ }^{1,9-11}$ If such a bump is incorporated in the functional form of the energy spectrum, realistic values for $\mathcal{S}_{3}$ can be obtained, ${ }^{12}$ in contrast to previous attempts discussed above. This is an important finding which, as discussed further below, we have also verified while investigating different candidate functional forms for the spectra: the inclusion of the bottleneck straightforwardly allows for realistic values of skewness of the longitudinal velocity derivative.

The parametrization of a bottleneck in the functional form for the energy spectrum necessarily introduces additional free parameters, which in the absence of an exact theory of turbulence must be fitted to experiments or DNS. In order to minimize the empiricism associated with such fits, one wishes to utilize "exact" constraints as much as possible. However, because there are more than two parameters required to include bottleneck and viscous effects, the parameters are not uniquely determined by the two constraints related to enstrophy and palinstrophy mentioned before. Additional parameters associated with the height, width, and location of the bottleneck are required. In the current study, we reduce the involved empiricism in selecting these parameters by introducing two new constraints. These constraints are based on the new observation that the height and location of the peak of the dissipation spectrum is inde- 
pendent of the Reynolds number, if intermittency corrections are included. To this end, a set of high-resolution DNS data for Reynolds numbers $\operatorname{Re}_{\lambda}$ between 70 and 1200 obtained from Ref. 10 and 11 is evaluated.

We propose a functional form for the energy spectrum which incorporates both the bottleneck and intermittency effects. The function contains five intrinsic parameters, of which four are related to the high-wavenumber adaptation of the inertial-range slope, and one to the low-wavenumber region. These parameters will be determined by five constraint relations. The first two were already given above and are supplemented with the requirement that the total energy,

$$
E_{t}=\int_{0}^{\infty} E(k) d k,
$$

which will mainly determine the low-wavenumber parameter. The location and the height of the intermittency corrected dissipation peak provide the two remaining conditions for the functional form of the spectrum. Using these five relations to solve for the parameters, we show that the proposed functional form fits DNS and experimental data very well over a wide range of Reynolds numbers. Since it is well known that the derivative skewness in turbulence displays a (weak) Reynolds number dependence, and there is possibly an intermittency correction to the spectral slope, the parameters characterizing the bottleneck and viscous decay will be shown to be by necessity (weakly) dependent on Reynolds number. Furthermore, different ranges will be shown to affect each other, e.g., the bottleneck range and the highwavenumber exponential decay display significant overlap. This makes analytical solutions impossible and requires the use of a numerical approach to find solutions to the set of coupled constraint equations.

In Sec. II, the functional form is introduced and the requirements related to energy, enstrophy, and palinstrophy are briefly reviewed. Subsequently, in Sec. III, observations on the intermittency corrected bottleneck are discussed, leading to two extra constraints for the functional form. In Secs. IV and V, results are discussed and comparisons with DNS and experimental data are shown. Conclusions are presented in Sec. VI.

This paper will not address the dynamical origins of the bottleneck, but it is mentioned that recent numerical experiments solving Navier-Stokes with hyperviscosity of various orders suggests that the bottleneck is due to "aborted thermalization," 13 i.e., a kind of "pileup" of energy near a cutoff scale when modes are not available at larger wavenumbers.

\section{FUNCTIONAL FORM}

First of all, following the classical conventions, we define a functional form for the energy spectrum as

$$
E(k)=C_{K} \varepsilon^{2 / 3} k^{-5 / 3}(k L)^{-\beta} f_{L}(k L) f_{\eta}(k \eta),
$$

where $C_{K}$ is the Kolmogorov constant, $k$ is the wavenumber, $\varepsilon$ is the rate of turbulent kinetic energy dissipation, and $\beta$ is the intermittency correction for the inertial-range slope of the energy spectrum. The large-scale length scale $L$ is defined as
$L \equiv E_{t}^{3 / 2} / \varepsilon$, and the Reynolds number $\operatorname{Re} \equiv E_{t}^{1 / 2} L / \nu$, such that $\operatorname{Re}=(L / \eta)^{4 / 3}$ when $\eta=\left(\nu^{3} / \varepsilon\right)^{1 / 4}$ is the Kolmogorov scale.

For the intermittency correction $\beta$ of the spectral slope, we take the log-normal approximation ${ }^{14} \beta=\mu / 9$, which should be reasonably accurate for the second order structure functions and the related energy spectra. ${ }^{15}$ The standard empirical value of $\mu=0.25$ for the intermittency exponent is a good representative value of most of the available literature. ${ }^{8}$

Following Pope's notation, ${ }^{8} f_{L}(k L)$ is a function which reshapes the $k^{-5 / 3}$ slope in wave numbers around the integral length scale $L$ [with $f_{L}(x \rightarrow \infty) \rightarrow 1$ and $f_{L}(x \rightarrow 0) \rightarrow 0$ ], while $f_{\eta}(k \eta)$ reshapes the spectrum near the Kolmogorov scale $\eta$ [with $f_{\eta}(x \rightarrow 0) \rightarrow 1$ and $f_{\eta}(x \rightarrow \infty) \rightarrow 0$ ]. We propose

$$
f_{\eta}(k \eta)=\exp \left(-\alpha_{1} k \eta\right)\left[1+\frac{\alpha_{2}\left(k \eta / \alpha_{4}\right)^{\alpha_{3}}}{1+\left(k \eta / \alpha_{4}\right)^{\alpha_{3}}}\right]
$$

where the factor

$$
B(k \eta)=\left[1+\frac{\alpha_{2}\left(k \eta / \alpha_{4}\right)^{\alpha_{3}}}{1+\left(k \eta / \alpha_{4}\right)^{\alpha_{3}}}\right]
$$

is the bottleneck correction, which is used to shape the spectral bump at the onset of the spectrum's dissipation range. We investigated different options for $B(k \eta)$, with amongst others a change of spectral slope,${ }^{16}$ or a construction using an $\arctan [\log (k \eta)]$ function. ${ }^{1,17}$ Among the options considered, we found Eq. (3) as the only form which could comply with the five constraints and retain satisfactory fits for the DNS and experiments. Consequently, in the current study, we will only present results which are constructed based on Eq. (3). Note that Eq. (3) was obtained by reconstructing the Cerruti and Meneveau ${ }^{17}$ form with a tanh instead of an arctan function. Also note that the approach in Refs. 1 and 17 was more ad hoc since they used direct fits to data to find all parameters without using constraint equations.

Our main focuss will be on the high-wavenumber part of the spectrum and $f_{\eta}$. However, for the comparison with experiments, a low-wavenumber function $f_{L}$ is also needed. To this end, we presume a Saffman spectrum $\left[E(k) \sim k^{2}\right]$ at low wavenumbers and use Von Kármán's interpolation function to obtain

$$
f_{L}(k L)=\left\{\frac{k L}{\left[(k L)^{p}+\alpha_{5}\right]^{1 / p}}\right\}^{5 / 3+\beta+2} .
$$

For the power $p$, a value of $p=1.5$ is employed. The functions $f_{L}$ and $f_{\eta}$ thus contain in total five parameters, $\alpha_{1}-\alpha_{5}$, for which five constraints will be formulated.

To this end, it is useful to define a compensated normalized spectrum as function of $k \eta$ according to

$$
F(k \eta)=\frac{k^{5 / 3}(k L)^{\beta} E(k)}{\varepsilon^{2 / 3}}=C_{K} f_{L}\left(k \eta \operatorname{Re}^{3 / 4}\right) f_{\eta}(k \eta) .
$$

Using this representation, the three conditions $E_{t}=\int E(k) d k$, $\mathcal{E}=\int k^{2} E(k) d k$, and $\mathcal{P}=\int k^{4} E(k) d k$ may be now, respectively, rewritten as ${ }^{2,6}$

$$
\int_{0}^{\infty} x^{-5 / 3-\beta} F\left(x \mathrm{Re}^{-3 / 4}\right) d x=1,
$$




$$
\begin{aligned}
& \int_{0}^{\infty} x^{1 / 3-\beta} \operatorname{Re}^{-3 \beta / 4} F(x) d x=\frac{1}{2}, \\
& \int_{0}^{\infty} x^{7 / 3-\beta} \operatorname{Re}^{-3 \beta / 4} F(x) d x=\frac{-7 \mathcal{S}_{3}}{12 \sqrt{15}},
\end{aligned}
$$

using $x=k \eta$ as an auxiliary variable.

It is easily verified that Eqs. (6)-(8) grow independent for increasing Reynolds numbers. In this case, the first [Eq. (6)] will only condition the shape of $f_{L}$, while the latter two will independently condition the shape of $f_{\eta}$. In a first step of the analysis, we will concentrate on the form of the viscous range and bottleneck function $f_{\eta}$ at high Reynolds numbers and presume that $f_{L}=1$, so that $F=C_{K} f_{\eta}$. As a consequence, condition (6) falls away, together with the need to find $\alpha_{5}$. Four parameters $\alpha_{1}-\alpha_{4}$ remain to be determined and two additional constraints besides Eqs. (7) and (8) are required. In order to motivate such constraints, in the next section, we attempt to collapse the viscous and bottleneck portions of the energy spectra obtained from DNS (Refs. 10 and 11) at various Reynolds numbers.

\section{INTERMITTENCY AND BOTTLENECK}

In order to high-light the high-wavenumber portion of the spectrum, we focus here on the dissipation spectrum $k^{2} E(k)$. Its integral is $\varepsilon / 2 \nu$. When nondimensionalized and written in Kolmogorov units, i.e., in terms of the variable $k \eta$, the dissipation spectrum can be written as $D(k \eta)$ $=(k \eta)^{1 / 3-\beta} \operatorname{Re}^{-3 \beta / 4} F(k \eta)$, i.e., the integrand in Eq. (7) which integrates out to $\frac{1}{2}$. Using the DNS data of Ref. 11, we evaluate $D(k \eta)$ and show the results in Fig. 1(a) at different Reynolds numbers. Clearly, these spectra depend on the Reynolds number, as can be observed in the height of the dissipation peak. Therefore, if one were to attempt fitting a bottleneck function, the height will be dependent on Reynolds number making the parametrization difficult. In Fig. 1(b), we plot $\operatorname{Re}^{3 \beta / 4} D(k \eta)$. As is apparent, in such an "intermittency corrected" scaling, the peak becomes much more independent of Reynolds number and a better collapse is observed. In particular, the peak values of the spectra coincide for DNS from two different sources ${ }^{11,10}$ and a wide range of Reynolds numbers between $\operatorname{Re}_{\lambda}=70$ and 1200. It is worth noting that experiments in shear flow (see Ref. 18) have shown the location of the dissipation peak to be independent of Reynolds number for $\operatorname{Re}_{\lambda}>200$. For $\operatorname{Re}_{\lambda}<200$ in those experiments with mean shear, the peak location increases with decreasing Reynolds number. This is in contrast to the almost constant peak location obtained from the forced DNS results ${ }^{10}$ (without shear) at $\operatorname{Re}_{\lambda}=70$ and 125 in Fig. 1. In the current study, we will mainly focus on $\operatorname{Re}_{\lambda}>200$ and exclude low Reynolds number effects. At low Reynolds numbers, differences between forced and decaying turbulence might introduce additional complications (recall that our enstrophy equilibrium constraint assumes stationarity or high Reynolds number).

Before proceeding, some counterintuitive trends need to be discussed. By looking at Eq. (7), we see that even in the presence of intermittency, the integral of $D(x)$ should be in-

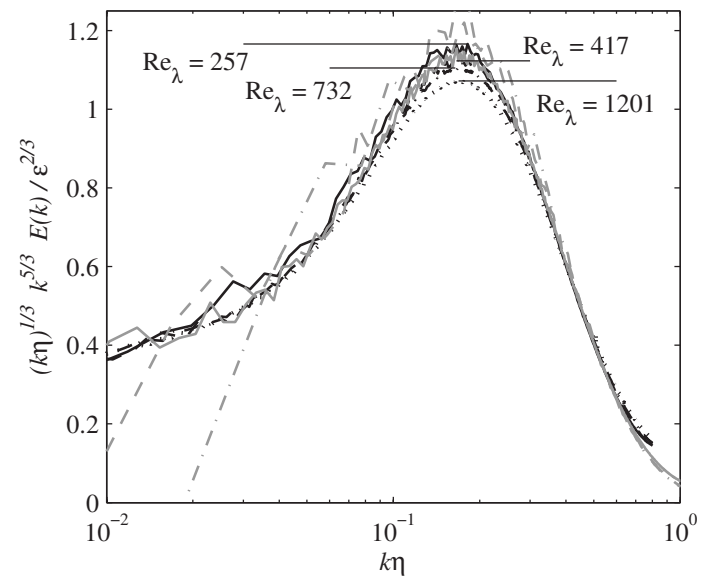

(a)

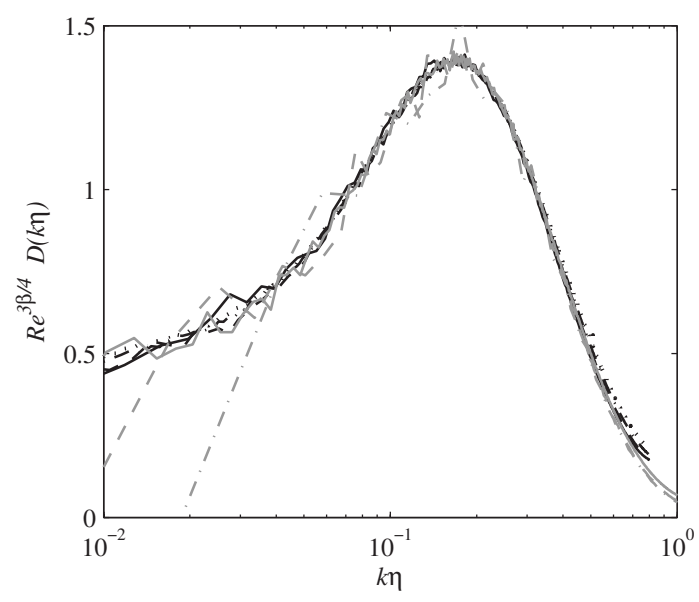

(b)

FIG. 1. Enstrophy spectra $D(k \eta)=(k \eta)^{1 / 3-\beta} \operatorname{Re}^{-3 \beta / 4} F(k \eta)$ (a) and $D(k \eta)$ multiplied with $\operatorname{Re}^{3 \beta / 4}$ (b). Black lines $(-;--;-\cdot ; \cdots) \operatorname{Re}_{\lambda}=257,417,732$, 1201 from DNS of forced isotropic turbulence by Kaneda et al. (Ref. 11). Gray lines (-; - -; -.) $\operatorname{Re}_{\lambda}=381,125,70$ from DNS of forced isotropic turbulence by Gotoh et al. (Ref. 10). In (a), the peak levels of the data of Kaneda et al. are marked.

dependent of Reynolds number, while $F(x)$ cannot be independent of Reynolds number since the left-hand side contains a factor $\mathrm{Re}^{-3 \beta / 4}$. However, in Fig. 1(a), it was shown that $D(x)$ clearly depends on the Reynolds number, as could be observed in the height of the dissipation peak, while as argued above its integral should be independent of the Reynolds number. Conversely, by the same argument, the spectra shown in Fig. 1(b), $\operatorname{Re}^{3 \beta / 4} D(x)$, should have a Reynoldsnumber-dependent integral (i.e., $\sim \operatorname{Re}^{3 \beta / 4}$ ). Yet, in the figure, these functions appear to be independent of the Reynolds number over a wide range of Reynolds numbers between $\operatorname{Re}_{\lambda}=70$ and 1200. The apparent contradiction can be resolved by evaluating the premultiplied spectrum $k \eta D(k \eta)$ in Fig. 2. The visual area under these curves (in semilog scale) is proportional to the value of the integral in Eq. (7), and from Fig. 2 there is indeed a dependence on the Reynolds number of $\operatorname{Re}^{3 \beta / 4} D(k \eta)$ coming from the high wavenumbers to the right of the peak. Therefore, there is no contradiction with the constraints. Subtle Reynolds number dependencies in the functional forms cannot be avoided.

The one robust feature that appears to be quite clearly independent of Reynolds number in Fig. 1(b) is the location 


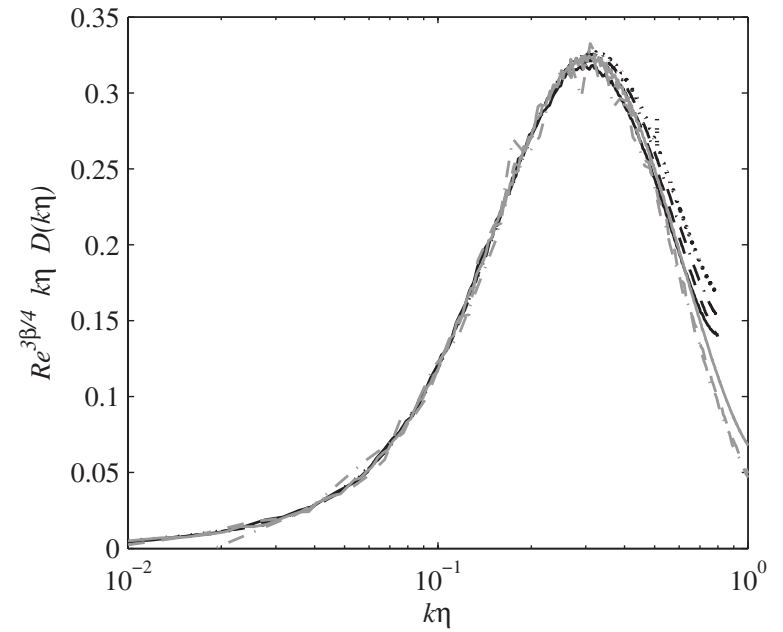

FIG. 2. Log-linear variance-proportional enstrophy spectra $k \eta D(k \eta)$ multiplied with $\mathrm{Re}^{3 \beta / 4}$. Line styles are the same as in Fig. 1.

and the height of the peak of the intermittency corrected dissipation spectrum $\operatorname{Re}^{3 \beta / 4} D(k \eta)$. Therefore, these two quantitative observations are chosen as the two extra conditions for the description of the energy spectrum, supplementing Eqs. (7) and (8). The location and the height are obtained from one of the cases, namely, the $1024^{3} \mathrm{DNS}$ at $\mathrm{Re}_{\lambda}=381$ from Ref. 10. The particular case is selected for its rather high resolution for the selected Reynolds number, such that also the palinstrophy spectrum, related to the calibration of $\mathcal{S}_{3}$, is well resolved.

From the data, we find that the peak occurs at $k_{p} \eta=0.167$ and that the peak has a height equal to $\operatorname{Re}^{3 \beta / 4} D\left(k_{p} \eta\right)=1.40$. This is consistent with visual observation in Fig. 1(b) but is obtained more precisely by using a calibration that includes fitting all parameters. The fit is obtained by optimizing the parameter set $\alpha_{1}-\alpha_{4}$ and $C_{k}$ such that the $L_{2}$-error between the $k^{5 / 3}$-compensated energy spectrum of the DNS and the functional form is minimal. Equation (7) is enforced as a constraint during the optimization. In contrast, for the calibration, Eq. (8) is only used afterward to obtain the skewness since $\mathcal{S}_{3}$ does not appear as an explicit parameter in the functional form (5). The constrained optimization problem is solved based on a sequential quadratic programing algorithm, for which the fmincon function of MATLAB was used. The fit yields that $C_{K}=2.0$ and $\alpha_{1} \approx 4.7$, $\alpha_{2} \approx 2.4, \alpha_{3} \approx 2.4$, and $\alpha_{4} \approx 0.12$. When evaluating the implied skewness coefficient, we obtain $\mathcal{S}_{3}=-0.58$, which corresponds well with the value of -0.574 listed in Ref. 10 .

In Fig. 3, the results of the calibration are shown in terms of the energy spectrum (instead of dissipation spectrum), and a very good agreement between the DNS data and the fitting function are found. Some thoughts have to be spent on the Kolmogorov constant $C_{K}$, found to be 2.0 in the calibration, which is considerably higher than an often quoted value of 1.6-1.7. The latter value has typically been obtained by evaluating $k^{5 / 3}$ premultiplied energy spectra and examining the plateau in some range of wavenumbers in the inertial range. For the sake of discussion, let us denote a representative wavenumber in this range as $k_{\mathrm{IR}}$. The resulting

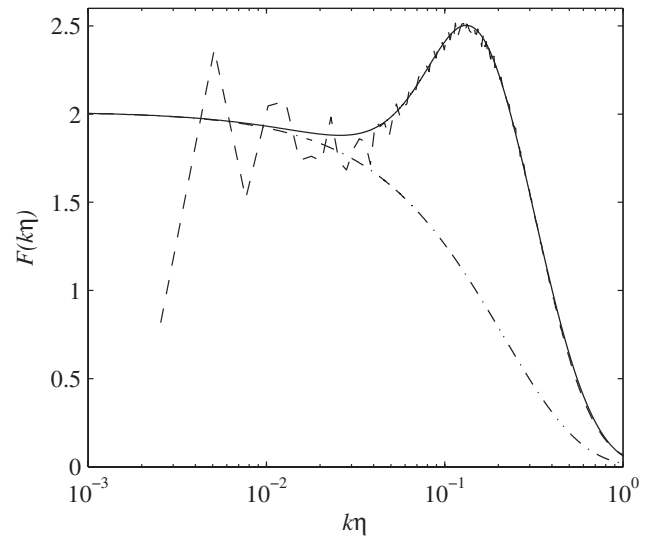

FIG. 3. Compensated spectrum $F(k \eta)$. (- -) Spectrum from a $1024^{3}$ DNS (Ref. 10) at $\operatorname{Re}_{\lambda}=381$; (-) fit based on Eq. (2); (-.) $C_{K} \exp \left(-\alpha_{1} k \eta\right)$, i.e., the same fit based on Eq. (2) but without the bottleneck factor of Eq. (3).

constant $C_{K}^{\prime}$ found from a classical $k^{5 / 3}$-compensation can be expressed as function of the constant $C_{K}$ in the current study by

$$
C_{K}^{\prime}=C_{K}\left(k_{\mathrm{IR}} L\right)^{-\beta} f_{\eta}\left(k_{\mathrm{IR}} \eta\right)
$$

Obviously, if intermittency plays a role, the factor $\left(k_{\mathrm{IR}} L\right)^{-\beta}$ can significantly influence the value found for $C_{K}^{\prime}$, especially at very high Reynolds numbers where an inertial-range wavenumber can be much larger than the wavenumber $L^{-1}$ corresponding to the integral scale (so that $k_{\mathrm{IR}} L \gg 1$ ). Moreover, $f_{\eta}\left(k_{\mathrm{IR}} \eta\right) \neq 1$ may also prompt differences between $C_{K}^{\prime}$ and $C_{K}$. This has been a known cause for an overestimation of the Kolmogorov constant in early DNS, where the constant was measured near the peak of the spectral bump ${ }^{19}$ such that $f_{\eta}>1$.

Prior to the predissipative bump in the spectrum, there may be a small dent in the compensated spectrum (with $f_{\eta}<1$ ), further complicating the measurement of the Kolmogorov constant. This dent was first highlighted in Ref. 20 based on atmospheric data at $\operatorname{Re}_{\lambda}=1.7 \times 10^{4}$. It was observed that the intermittency corrected slope $(-5 / 3-\beta)$ was only recovered for $k_{1} \eta<2 \times 10^{-3}$ (in a one-dimensional spectrum), while prior to the bottleneck, a steepening of the slope was observed. In the calibration of Fig. 3, such a dent is also observed. Here, this dent is related to the early onset of the exponential decay.

\section{BEHAVIOR AT OTHER REYNOLDS NUMBERS}

In this section, four constraints are applied for a variety of Reynolds numbers and the results are compared with DNS. Effects related to the fifth parameter and constraint and the low-wavenumber part of the spectrum are discussed in the next section.

The constraint involving the palinstrophy requires the knowledge of the skewness coefficient $\mathcal{S}_{3}$. Following standard intermittency estimates, ${ }^{15}$ a Reynolds dependent skewness $\mathcal{S}_{3}$ is prescribed according to 
TABLE I. Overview of $\alpha_{1-5}$ for different cases. The reference case used for the fit of $C_{K}$ and the height and location of the intermittency-corrected dissipation peak (cf. Fig. 3) is displayed in italic.

\begin{tabular}{lccccccc}
\hline \hline $\operatorname{Re}_{\lambda}$ & Ref. & Fig. & $\alpha_{1}$ & $\alpha_{2}$ & $\alpha_{3}$ & $\alpha_{4}$ & $\alpha_{5}$ \\
\hline 257 & DNS $^{\mathrm{a}}$ & 4 & 4.872 & 2.630 & 2.292 & 0.1275 & $\ldots$ \\
381 & DNS $^{\mathrm{b}}$ & 3 & 4.677 & 2.365 & 2.439 & 0.1212 & $\ldots$ \\
417 & $\mathrm{DNS}^{\mathrm{a}}$ & 4 & 4.635 & 2.312 & 2.475 & 0.1200 & $\ldots$ \\
732 & $\mathrm{DNS}^{\mathrm{a}}$ & 4 & 4.387 & 2.023 & 2.733 & 0.1138 & $\ldots$ \\
1201 & $\mathrm{DNS}^{\mathrm{a}}$ & 4 & 4.190 & 1.822 & 3.013 & 0.1101 & $\ldots$ \\
250 & $\ldots$ & $\ldots$ & 4.972 & 2.957 & 2.019 & 0.1385 & 3.967 \\
500 & $\ldots$ & $\ldots$ & 4.569 & 2.261 & 2.457 & 0.1190 & 4.068 \\
716 & Expt. $^{\mathrm{c}}$ & 5 & 4.404 & 2.054 & 2.665 & 0.1144 & 4.136 \\
750 & $\ldots$ & $\ldots$ & 4.384 & 2.031 & 2.693 & 0.1139 & 4.145 \\
1000 & $\ldots$ & $\ldots$ & 4.265 & 1.901 & 2.868 & 0.1114 & 4.192 \\
1250 & $\ldots$ & $\ldots$ & 4.177 & 1.813 & 3.013 & 0.1100 & 4.224 \\
\hline$\overline{\mathrm{a}}$ & $\ldots$ & &
\end{tabular}

${ }^{\mathrm{a}}$ Reference 11 .

${ }^{\mathrm{b}}$ Reference 10

${ }^{c}$ Reference 1.

$$
\mathcal{S}_{3}=C_{3} \mathrm{Re}_{\lambda}^{\xi},
$$

where $C_{3}$ is a constant and $\xi$ is an exponent which can be determined to be ${ }^{21} \xi=9 \mu / 16$. The constant $C_{3}$ is inferred from the calibrated reference value of $\mathcal{S}_{3}=-0.58$ at $\operatorname{Re}_{\lambda}=381$, namely, $C_{3}=-0.25$.

Finally, the four constraints now consist of Eqs. (7) and (8), complemented with conditions for the height and location of the peak of $\operatorname{Re}^{3 \beta / 4} D(x)=x^{1 / 3-\beta} F(x)$ [cf. Fig. 1(b)]. Combining with Eq. (5) and canceling $C_{K}=2.0$ leads to

$$
\begin{aligned}
& \left.\frac{d}{d x}\left[x^{1 / 3-\beta} f_{L}\left(x \operatorname{Re}^{3 / 4}\right) f_{\eta}(x)\right]\right|_{x=0.167}=0, \\
& \left.x^{1 / 3-\beta} f_{L}\left(x \operatorname{Re}^{3 / 4}\right) f_{\eta}(x)\right|_{x=0.167}=0.70 .
\end{aligned}
$$

Here, $f_{\eta}(x)$ is given by Eq. (2) (dependent on parameters $\alpha_{1}-\alpha_{4}$ ), while $\mathcal{S}_{3}$ is given by Eq. (10), and $\beta=\mu / 9$. Further, for the analysis in the current section, $f_{L}=1$, i.e., the lowwavenumber part of the spectrum is not included. The parameters $C_{K}=2.0, C_{3}=-0.25$, and $\mu=0.25$ are held fixed (independent of $\mathrm{Re}$ ), while the values of parameters $\alpha_{1}-\alpha_{4}$ are obtained from solving the above four constraints. To this end, we employ the fsolve function of MATLAB, which uses a trust-region dogleg method to solve systems of nonlinear equations. For the numerical evaluation of the integrals in Eqs. (7) and (8), adaptive Lobatto quadrature is used, for which standard functions are also available in MATLAB.

In Table I an overview is presented of the coefficients obtained by solving these equations for the different cases. As can be observed from the table, the coefficients $\alpha_{1-4}$ vary slowly and monotonously with $\operatorname{Re}_{\lambda}$, such that for practical use, simple interpolation of results in this table could be adequate to find values at intermediate Reynolds numbers. Here, we refrain from proposing such interpolation, instead suggesting that users simply solve the constraint equations for any desired Reynolds number.

In Fig. 4, the resulting functional forms are presented for $\operatorname{Re}_{\lambda}=257,417,732,1201$. As comparison, DNS spectra from forced homogeneous isotropic turbulence at the same Reynolds numbers are also displayed, ${ }^{11}$ and very good agreement is observed.

\section{BEHAVIOR AT LOW $k$ AND COMPARISON WITH EXPERIMENTS}

Until now, the low-wavenumber correction of the inertial-range spectrum [Eq. (4)] and its associated parameter $\alpha_{5}$ were not used. The use of the representation of the lowwavenumber modes in DNS of forced isotropic turbulence is not relevant. However, for the representation of experimental data with a significant infrared spectrum, $f_{L}[\mathrm{Eq}$. (4)] is useful. In Table I an overview is also presented of the coefficients $\alpha_{1-5}$ obtained by solving the constraints now also including Eq. (6) [i.e., $\int_{0}^{\infty} x^{-5 / 3-\beta} C_{K} f_{L}(x) f_{\eta}\left(x \mathrm{Re}^{-3 / 4}\right) d x=1$ ] for different Reynolds numbers. As commented before in Sec. II, the low- and high-wavenumber functions $f_{L}$ and $f_{\eta}$ grow mutually independent for increasing Reynolds numbers. For

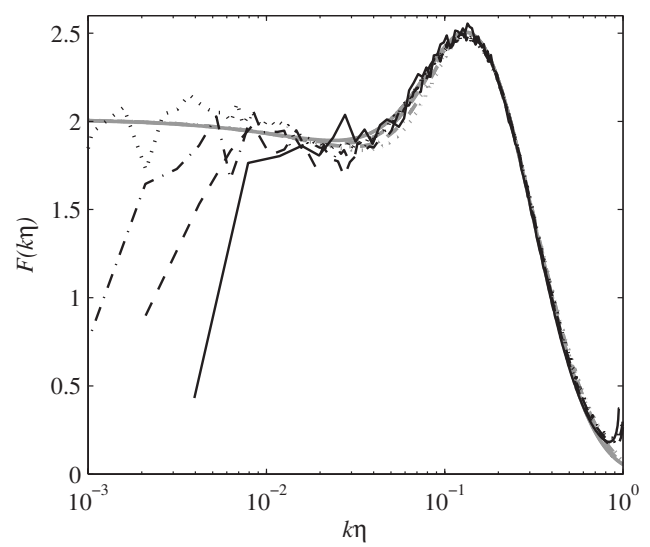

FIG. 4. Compensated spectrum $F(k \eta)=\varepsilon^{-2 / 3} k^{5 / 3}(k L)^{\beta} E(k)$. (-; - -; -; ; ) $\operatorname{Re}_{\lambda}=257,417,732$, 1201. (In black) DNS of forced isotropic turbulence by Kaneda et al. (Ref. 11) (In gray) Fitted functional forms $F(k \eta)$ $=C_{K} f_{L}\left(k \eta \operatorname{Re}^{3 / 4}\right) f_{\eta}(k \eta)$ for the spectrum. 


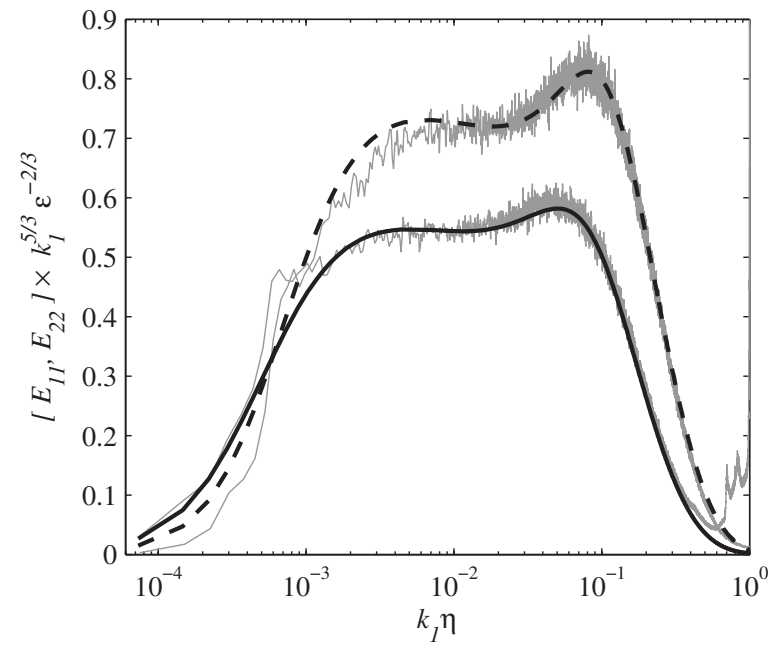

FIG. 5. $k^{5 / 3}$-compensated one-dimensional spectra $E_{11}$ and $E_{22}$. (In black) (-) $E_{11}$ (compensated) based on the functional form [Eqs. (1), (2), and (4)]; $(--), E_{22}$ (compensated) based on Eqs. (1), (2), and (4). (In gray) Experimental data at $\operatorname{Re}_{\lambda}=716$ from Kang et al. (Ref. 1).

$\operatorname{Re}_{\lambda}=500$, the largest deviation we observed for $\alpha_{1-4}$ between cases with and without $\alpha_{5}$ is $3 \%$. For $\operatorname{Re}_{\lambda}=750$, this difference further reduced to $1 \%$.

We now turn to the active grid turbulence of Kang et al., ${ }^{1}$ using data from the first measurement station $(x / M$ =20) with $\operatorname{Re}_{\lambda}=716$. Results of one-dimensional spectra are presented in Fig. 5. For the functional form, the onedimensional spectrum is obtained from the three-dimensional spectrum [Eq. (1)] using ${ }^{8} E_{11}\left(k_{1}\right)=\int_{1}^{\infty}\left(y^{2}-1\right) / y^{3} E\left(k_{1} y\right) d y$, with the auxiliary variable $y=k / k_{1}$. Similarly, $E_{22}\left(k_{1}\right)$ $=\frac{1}{2} \int_{1}^{\infty}\left(y^{2}+1\right) / y^{3} E\left(k_{1} y\right) d y$. These integrations are straightforward to perform using numerical integration for a large set of $k_{1}$ values.

Since the Kolmogorov scale in the experiments was under resolved, ${ }^{1}$ the experimental uncertainty for the dissipation is about $10 \%$. The experimental data in Fig. 5 are normalized using $\varepsilon=19.7 \mathrm{~m}^{2} / \mathrm{s}^{3}$, which is about $10 \%$ lower than the estimated value reported in Ref. 1. Apart from this uncertainty on the level of the compensated experimental spectra, it may be appreciated that the shape of the functional representation fits the experimental data very well. Similar results (not shown here) are obtained for the data of Kang et $a l$. at downstream measurement stations.

In order to explore the limitations of our proposed functional form, we try to represent the atmospheric data of Tsuji ${ }^{20}$ obtained at $\operatorname{Re}_{\lambda}=17060$. These data are at a Reynolds number $\operatorname{Re}_{\lambda}$ which is a factor 45 higher (and a factor $\sim 2000$ based on Re) than the DNS data which were used to calibrate $C_{K}, C_{3}$, and Eq. (11) in Sec. III. Unfortunately, we find that the use of $C_{K}=2.0$ combined with Eq. (10) (which would yield $\mathcal{S}_{3}=-0.99$ ) does not straightforwardly lead to a good representation of the experimental data. In Fig. 6, we present a functional form with $C_{K}=2.3$ and $\mathcal{S}_{3}=-0.86$, but further using Eqs. (6)-(8) and (11) and this leads to a satisfactory fit. The value $C_{K}=2.3$, which is used for the Kolmogorov constant, is in good agreement with Tsuji's data: by taking into account Eq. (9), we find that the value $C_{K}=2.3$ corresponds to a one-dimensional constant $C_{1 \mathrm{D}}=18 / 55 C_{K}^{\prime}=0.64$, which is

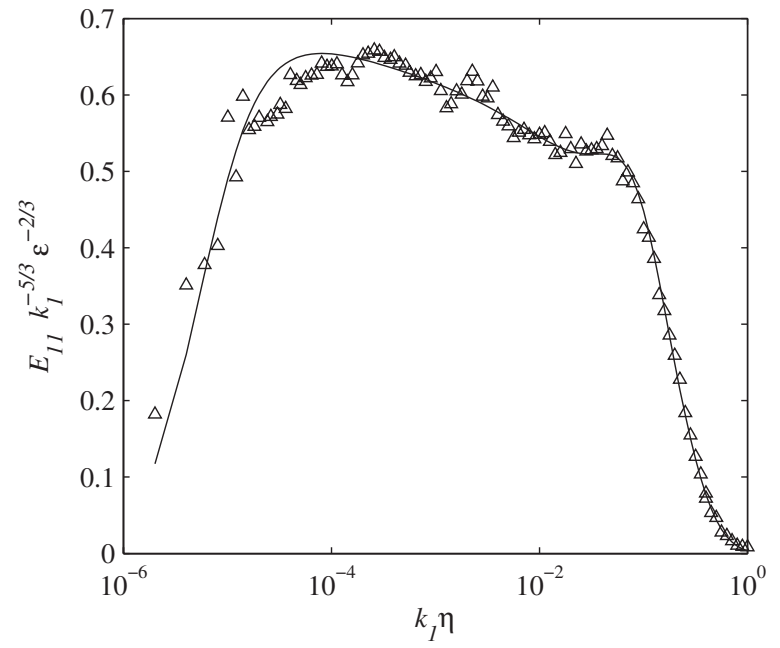

FIG. 6. $k^{5 / 3}$-compensated one-dimensional spectra $E_{11}$. (-) $E_{11}$ (compensated) based on the functional form [Eqs. (1), (2), and (4)]; $\triangle$ ) experimental data at $\operatorname{Re}_{\lambda}=17060$ from Tsuji (Ref. 20).

close to the value reported in Ref. 20. However, for $\mathcal{S}_{3}$, no results were reported so no direct comparison can be done.

Though results in Fig. 6 demonstrate that the functional form described in Sec. II can also be used to appropriately represent atmospheric data at very high Reynolds numbers, this was not achieved by straightforwardly carrying over the value for $C_{K}$ and $\mathcal{S}_{3}$ obtained from Eq. (10). Clearly, this asks for further research.

\section{CONCLUSIONS}

In the current work, we evaluated a new functional form for the energy spectrum of high Reynolds number turbulence, which accounts for bottleneck and intermittency effects. Central to a generic parametrization was the observation that the peaks of intermittency corrected dissipation spectra collapse for a large set of DNS data with Reynolds numbers $\operatorname{Re}_{\lambda}$ between 70 and 1200 . This allowed us to state two constraints on the functional form; together with constraints on the total integrated energy, enstrophy (related to total dissipation), and palinstrophy (related to the skewness of the longitudinal velocity derivative), these form a set of five constraints, allowing a sufficiently flexible but welldefined formulation of the functional form. First, a calibration of the functional form was performed based on DNS data at $\operatorname{Re}_{\lambda}=381$. This yielded the reference level and location of the intermittency corrected dissipation peak, the Kolmogorov constant, and a reference value for the skewness $\mathcal{S}_{3}$. Subsequently, the functional form was evaluated at different Reynolds numbers. Moreover, in the spirit of an intermittency corrected energy spectrum, Nelkin's ${ }^{21}$ Reynolds-number dependence of the skewness was used, with the calibrated value at $\operatorname{Re}_{\lambda}=381$ as a reference. In this way, we compared the proposed parametrization of the energy spectrum with DNS and experimental data for $\mathrm{Re}_{\lambda}$ ranging from 250 to 1200. Excellent results were obtained for all these Reynolds numbers. Applications to data at much higher Reynolds numbers, however, displayed some discrepancies with the fitted parameter values. These difficulties show that 
further refinements of the functional form may be required for applicability at very high Reynolds numbers. This is also related to the longstanding question about universality of the Kolmogorov constant ${ }^{22}$ and to the applicability of approaches based on matched asymptotic expansions. ${ }^{23}$

Finally, for experimental determinations of the dissipation rate when the viscous scales cannot be resolved due to limited probe resolution, often the measured energy spectrum in the inertial range is used, coupled with assumptions of inertial-range scaling and assuming a universal value for $C_{K}$. According to Eq. (1), results presented in this paper suggest that one should also include the intermittency correction and the higher value of $C_{K} \sim 2.0$ when determining $\varepsilon$ from experimental energy spectra in the inertial range. Alternatively, the height of the intermittency corrected dissipation peak, given in Eq. (11), could also be used as a point of reference for the determination of $\varepsilon$ (as long as the peak can be resolved in experiments). However, the universality of $C_{K}$ remains an important issue for the reliability of such a method.

\section{ACKNOWLEDGMENTS}

We gratefully acknowledge Y. Kaneda and Y. Tsuji for sending us their data sets. We also thank G. Eyink for some useful discussions and B. J. Geurts for introducing the authors. We further acknowledge financial support from the Science Foundation-Flanders (FWO-Vlaanderen) for sponsoring the visit of J.M. at Johns Hopkins University. The research of C.M. is supported by the U.S. National Science Foundation. J.M. is a postdoctoral research fellow of the Research Foundation-Flanders (FWO-Vlaanderen), Belgium.

${ }^{1}$ H. S. Kang, S. Chester, and C. Meneveau, "Decaying turbulence in an active-grid-generated flow and comparisons with large-eddy simulation," J. Fluid Mech. 480, 129 (2003).

${ }^{2} \mathrm{~J}$. Meyers and M. Baelmans, "Determination of subfilter energy in largeeddy simulations," J. Turbul. 5, 26 (2004).

${ }^{3}$ J. O. Hinze, Turbulence, 2nd ed., McGraw-Hill Series in Mechanical Engineering (McGraw-Hill, 1975).
${ }^{4}$ R. H. Kraichnan, "The structure of isotropic turbulence at very high Reynolds numbers," J. Fluid Mech. 5, 497 (1959).

${ }^{5}$ S. Chen, G. Doolen, J. R. Herring, R. H. Kraichnan, S. A. Orszag, and S. S. Zhen, "Far dissipation range of turbulence," Phys. Rev. Lett. 70, 3051 (1993).

${ }^{6}$ L. M. Smith and W. C. Reynolds, "The dissipation-range spectrum and the velocity-derivative skewness in turbulent flows," Phys. Fluids A 3, 992 (1991).

${ }^{7}$ T. Sanada, "Comment on the dissipation-range spectrum in turbulent flows," Phys. Fluids A 4, 1086 (1992).

${ }^{8}$ S. B. Pope, Turbulent Flows (Cambridge University Press, Cambridge, 2000).

${ }^{9}$ S. G. Saddoughi and S. V. Veeravalli, "Local isotropy in turbulent boundary layers at high Reynolds number," J. Fluid Mech. 268, 333 (1994).

${ }^{10}$ T. Gotoh, D. Fukayama, and T. Nakano, "Velocity field statistics in homogeneous steady turbulence obtained using a high-resolution direct numerical simulation," Phys. Fluids 14, 1065 (2002).

${ }^{11}$ Y. Kaneda, T. Ishihara, M. Yokokawa, K. Itakura, and A. Uno, "Energy dissipation rate and energy spectrum in high resolution direct numerical simulations of turbulence in a periodic box," Phys. Fluids 15, L21 (2003).

${ }^{12} \mathrm{M}$. Coantic and J.-J. Lasserre, "On pre-dissipative 'bumps' and a Reynolds-number-dependent spectral parameterization of turbulence," Eur. J. Mech. B/Fluids 18, 1027 (1999).

${ }^{13}$ U. Frisch, S. Kurien, R. Pandit, W. Pauls, S. S. Ray, A. Wirth, and J.-Z. Zhu "Hyperviscosity, Galerkin truncation and bottlenecks in turbulence," e-print arXiv:0803.4269.v2.

${ }^{14}$ A. N. Kolmogorov, "A refinement of previous hypotheses concerning the local structure of turbulence in a viscous incompressible fluid at high Reynolds number," J. Fluid Mech. 13, 82 (1962).

${ }^{15}$ U. Frisch, Turbulence (Cambridge University Press, Cambridge, 1995).

${ }^{16}$ Z.-S. She and E. Jackson, "On the universal form of energy spectra in fully developed turbulence," Phys. Fluids A 5, 1526 (1993).

${ }^{17} \mathrm{~S}$. Cerutti and C. Meneveau, "Statistics of filtered velocity in grid and wake turbulence," Phys. Fluids 12, 1143 (2000).

${ }^{18}$ Y. Tsuji, "Large-scale anisotropy effect on small-scale statistics over rough wall turbulent boundary layers," Phys. Fluids 15, 3816 (2003).

${ }^{19}$ P. K. Yeung and Y. Zhou, "Universality of the Kolmogorov constant in numerical simulations of turbulence," Phys. Rev. E 56, 1746 (1997).

${ }^{20} \mathrm{Y}$. Tsuji, "Intermittency effect on energy spectrum in high-Reynolds number turbulence," Phys. Fluids 16, L43 (2004).

${ }^{21}$ M. Nelkin, "Multifractal scaling of velocity derivatives in turbulence," Phys. Rev. A 42, 7226 (1990).

${ }^{22}$ K. R. Sreenivasan, "On the universality of the Kolmogorov constant," Phys. Fluids 7, 2778 (1995).

${ }^{23}$ T. S. Lundgren, "Kolmogorov turbulence by matched asymptotic expansions," Phys. Fluids 15, 1074 (2003). 